\title{
Therapy of Spine Metastasis Causing Paralysis Symptoms - Operation and Rehabilitation
}

\author{
Lars Homagk*, Pataraia A and Röhl K \\ Department for Spinal Cord Injuries, BG Kliniken Bergmannstrost Halle/Saale, Germany
}

\begin{abstract}
Most common manifestation of tumor metastasis after lungs and liver is the skeletal system with $60-80 \%$ of spine metastasis. In $30 \%$ of all cancer patients with metastatic spinal complaints are initial presentation of malignant primary disease while the primary paraplegia occurs in $5-10 \%$ of all spinal metastases. Thus a further operational metastases treatment is dependent on the entire metastasis status, the type and Tomita score as well as the risk of surgery considering general patient status. From 01.01 .11 to 31.12 .12 we included 16 patients with tumors. In $27 \%$ of the cases the first symptoms were paraplegia, but the primary tumor was determined in only one of these cases. $73 \%$ of patients underwent surgical treatment within the first 24 hours after admission. The hospital stay was 22 days. $56.3 \%$ of patients had incomplete paralysis at admission and $71 \%$ of these patients had postoperative improvement in neurological outcomes. All patients benefited as part of operational and rehabilitative treatment of a significant reduction in pain and $50 \%$ of patients were able to be discharged home. At the onset of paralytic symptoms we recommend immediate operation. In a multidisciplinary case conference the oncological treatment regimens should be defined also for the necessary paralysis treatment. Decision guidance is the height of paralysis, tumorgenesis, ASA classification and the pain symptoms of the patient. The treatment must adjusted and individualized according to the patient's general health, life expectancy, the primary tumor, the grading of metastasis and extent of neurological deficit.
\end{abstract}

\section{Introduction}

Tumors of the spine are primarily categorized by their location to the spinal cord. Thereby are intramedullary tumors with 5-10\% of spinal tumors rare. The intradural extramedullary tumors make $40 \%$ of spinal tumors and are often meningiomas or peripheral nerve sheath tumors. Extradural tumors and metastases are with $50-55 \%$ the most common spinal tumors [1]. The skeletal system is the lung and liver after the third most common manifestation of tumor metastases [2]. Thereby the spine with $60-80 \%$ represents the most common manifestation of localization. In almost one third of all cancer patients metastatic spinal complaints are the first manifestation of malignant primary disease [3]. These metastases usually are hematogenous and can be morphologically indicative of the primary tumor. The incidence of spinal metastases is dependent on the primary tumor, and with 60 $80 \%$ of the most frequent prostate tumor in men and up to $80 \%$ belongs to a mammary tumor in women. Other primary tumors are of the lung, kidney and thyroid. At 10\%, it cannot be found a primary tumor (CUP= carcinoma unknown primary) $[2,4]$. The hematogenous dissemination along with the lymphatic spread and spread by continuity is the most common way of metastatic spread [5]

Thus spinal metastases are a common complication of underlying malignant disease at an advanced stage. Due to demographic changes of our population not only the incidence of tumors, but also the paraplegia incidence rises due to spinal metastasis. A spinal cord injury is a complex, resulting from a complete or incomplete spinal cord injury, paralysis with loss of motor, sensitive and autonomic functions below the lesion. The classification and progression are based on ASIA scheme of the American Spinal Injury Association [6], which is based on the modified Frankel scale [7]. The primary symptoms of paraplegia may be the first manifestation of an underlying malignant disease and occurs with $5-10 \%$ of all spinal metastases. Here range the neurological deficits from sensory disturbances to a complete paraplegia [8]. Acute neurological deficits require rapid surgical decompression.

This paraplegia underlay a primary disease with a limited life expectancy. Their treatment is not only responsible for the spine surgeon.
The initial diagnosis of paraplegia is at first limited to the related causes. Nevertheless, the secondary tumor therapy has an important role in the treatment of these patients and their outcome $[9,10]$. In addition to the clinical neurological examination and a tumor-specific laboratory tests, the imaging methods have a high priority. The magnetic resonance imaging (MRI) represents the current method of choice in showing the number, size and spread of the metastases in the diagnosis and assessment of spinal tumors. Computed tomography (CT), if necessary myelography, serve to depict bone erosions, instability or fractures. The X-ray in 2 planes is particularly used in the postoperative control of instrumentation. As part of the diagnosis in the absence of surgical indication or capability can also a CT or ultrasound-assisted biopsy be performed $[11,12]$.

The first goal of the surgical therapy in case of additional paralysis symptoms is not only the complete removal of the tumor and the decompression of the neural structures, but also relief of pain and neurological deficits, as well as the obtaining positioning or exercise stability. Nevertheless, there should be an interdisciplinary preoperative assessment of benefit and risk of the procedure, particularly in patients with severe neurological deficits, multiple metastases or progressed cancer and a reduced overall prognosis. Classifications of severity and prognosis have been established by the Tomita or Tokuhashi score [13]. Therefore a further operational metastases treatment is dependent on the entire metastasis status, the Tomita-type and -score as well as

*Corresponding author: Dr. Lars Homagk, BG-Kliniken Bergmannstrost, Merseburger Str. 165, 06112 Halle/Saale, Germany, Tel: 0049 (0) 345 1327077; Fax: 0049 (0) 345 1326313; E-mail: Lars.Homagk@bergmannstrost.com

Received July 03, 2014; Accepted July 24, 2014; Published September 20, 2014

Citation: Homagk L, Pataraia A, Röhl K. Therapy of Spine Metastasis Causing Paralysis Symptoms - Operation and Rehabilitation. Journal of Surgery [Jurnalul de chirurgie] 2014; 10(2): 145-148 DOI: 10.7438/1584-9341-10-2-8

Copyright: (c) 2014 Homagk L, et al. This is an open-access article distributed under the terms of the Creative Commons Attribution License, which permits unrestricted use, distribution, and reproduction in any medium, provided the original author and source are credited. 
the risk of surgery considering the general patient status. Moreover, the temporary progression of neoplastic disease should be considered [11]. The surgical options range from decompression in prostate metastases or patients with very poor overall prognosis to combined ventrodorsal tumor resection with vertebral body replacement and stabilization. In case of bad Tomita score, a survival time of 12 months or reduced general health condition, the resection of the tumor is recommended only with stabilization. Other less invasive procedures are the augmenting of vertebral bodies with bone cement, a mono- or bisegmental laminectomy/laminoplasty or hemilaminectomy in small or lateralized tumors $[14,15]$.

\section{Material and Methods}

From 01.01 .11 to 31.12 .12 we have treated 104 acute spinal cord injuries. Therefrom 16 patients, at an average age of 67.1 years, were already tetra- ore paraplegic due to spinal metastasis or a primary tumor. Within the preoperative diagnosis according to the general condition of the patient were classified by the anesthesiologist using the ASA-classification. The ASA classification is widely used in medicine for classifying patients into different groups with respect to the physical condition $[16,17]$.

In addition to it the corresponding tumor score is classified after Tokuhashi and Tomita. Tokuhashi et al. designed 1994 a tumor score for spinal metastases, which takes into consideration the general condition of the patient, the number of extraspinal bone metastases, the number of metastases in the spine, the presence of metastases in internal organs, the primary tumor site and the severity of spinal cord injury. A maximum of 12 points can be obtained [13]. In 2001 Tomita et al. set up a strategy for the surgical treatment of spinal metastases. Their scoring system evaluates different prognostic factors and divides the expected therapeutic result into long-, medium-or short-term improvement of symptoms or terminal stage [18].

In the electronic medical records in our hospital, the pain intensity of inpatients are obtained and evaluated using the numeric rating scale (NRS). The further registration of pain intensity was performed daily, at rest, at expression of pain, at changes in the treatment of pain and at movement [19] (Figures 1 and 2).

\section{Results}

Due to an impending or present paraplegia, after neurological diagnosis, immediate computed tomography and magnetic resonance imaging were done. Surgical treatment with spinal decompression and spinal fusion was carried out until the next day after admission of patients. In $26.7 \%$ of cases was the paraplegia first symptom of a previously unknown tumor. The primary tumor could not be determined in 2 cases in the postoperative staging and vertebral body histology $(12.5 \%$, CUP). In the other cases it was a metastasis in at least for 1 year known primum. From the entity the genitourinary tract tumors were most common with $43.8 \%$. Specifically, there were 3

\section{Tomita score and ASA classification}

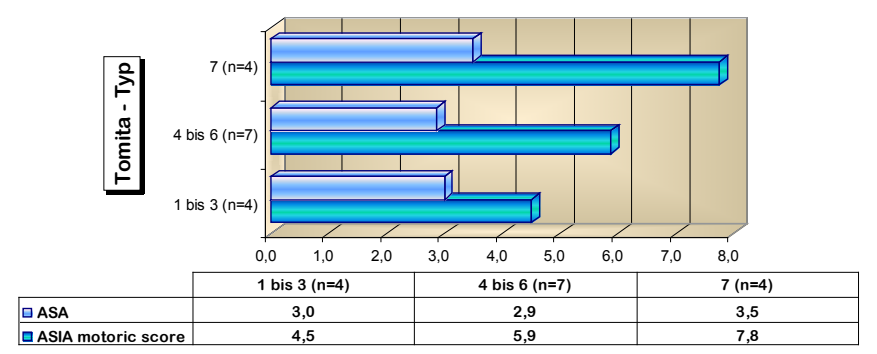

Figure 1: Distribution Tomita score and ASA classification by Tomita-type.

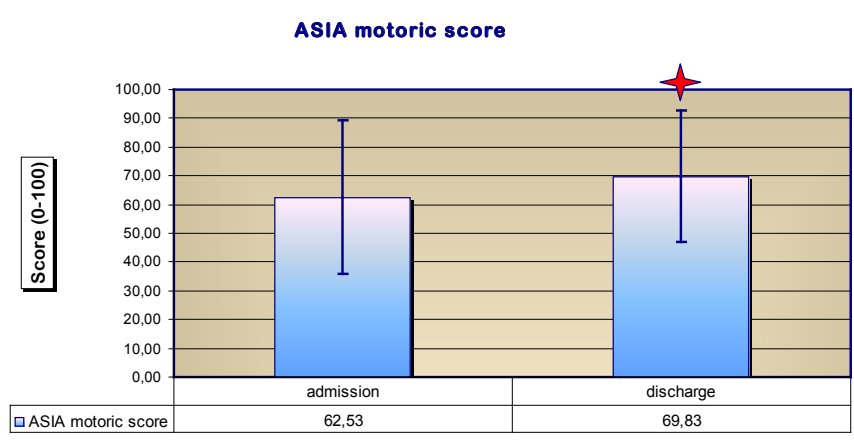

Figure 2: ASIA motor score during the inpatient treatment, ${ }^{*} p<0.1$.

prostate tumors and 3 renal cell carcinoma, as well as a Urethelkarzinom tumors. Other entities were breast, pancreatic cancers and lung cancer. A plasmacytoma and a Pancosttumor in NSCLC (non-small cell lung cancer) also led to paraplegia. The average hospital stay was 22.1 days with a minimum of 3 days and a maximum of 65 days.

At the time of admission, patients were due to the underlying disease in a reduced general condition. At the time of operative preparation by the anesthesiologists, the average ASA score was 3.2. 44\% patients were classified as ASA 4 . Nevertheless $73 \%$ of patients received surgical treatment within the first 24 hours. The localization of tumor was in $74 \%$ of the thoracic spine, in $16 \%$ cervical spine, and $11 \%$ in the lumbar spine. Respectively 4 cases were from Tomita-type 1-3 and 7. 8 cases were the Tomita type 4-6. The Tomita score of patients increased in the Tomita-type groups of 4.5 (Tomita-type 1-3) with 5.9 to 7.8 in Tomitatype 7. Simultaneously the same Tokuhashi score decreased from 7.0 to 3.8 or 4.5 . The correlation of this classification scores with the general condition of the patient shows the proportional increasing of the ASA classification in rising Tomita score and -type.

13 of 15 patients underwent a surgery with decompression of spinal canal and internal fixation after preoperative diagnostic. One patient died before surgery because of poor health. In total 3 Patients died during the inpatient stay (18.8\%).

At the admission 4 patients had no motor deficits (ASIA E) and could be discharged without neurological disorders. 3 patients were complete plegic (ASIA A) and showed no motor recovery. At the admission $56.3 \%$ of patients had incomplete paralysis (ASIA BD). $71 \%$ of these patients showed a postoperative improvement in neurological outcomes. 3 patients converted and improved from ASIA B to C and C to D. The ASIA motor scores of the 5 extremity muscles in this group of patients improved from 54 points to 67 points and in all patients from 62 to 70 points.

$94 \%$ of patients were transferred to our center for secondary diagnostics and treatment from the other hospitals. Nevertheless, all patients benefited in a significant reduction of pain after operational and rehabilitative treatment.

After an acute-treatment, rehabilitation and an oncological aftercare, due to consensus of an interdisciplinary tumor boards, were initiated. $50 \%$ of patients could be discharged to follow-up outpatient treatment. 2 patients were transferred for further oncological treatment in hospitals nearer to their homes. 2 patients were discharged directly into the inpatient rehabilitative follow-up and 1 patient in a hospice near home (Figures 3 and 4).

Due to the reduced general condition of the patient and the underlying primary disease the higher rate of complications is to be expected. From the same patient population 2 patients died postoperatively, and 2 patients had to be re-operated because of an early surgical termination and a wound infection. 2 patients occurred 
Pain intensity

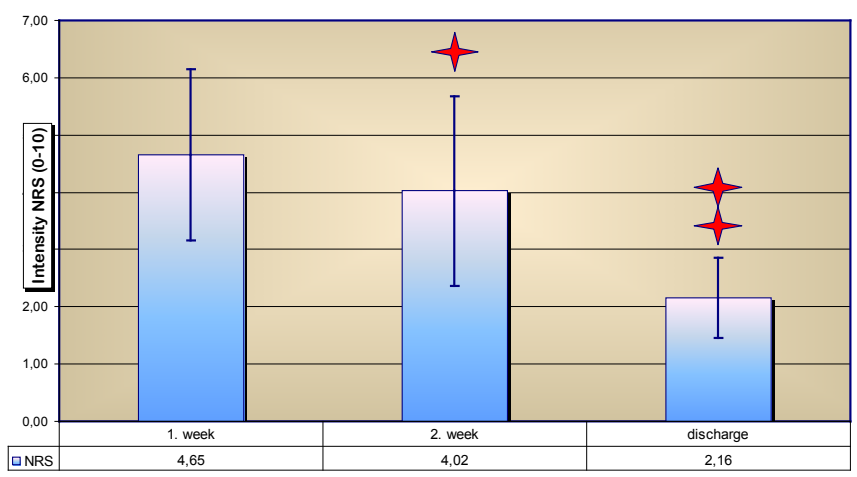

Figure 3: Pain intensity during the course of hospitalization, ${ }^{*}=p<0.05$.

\section{Transferring statstics}

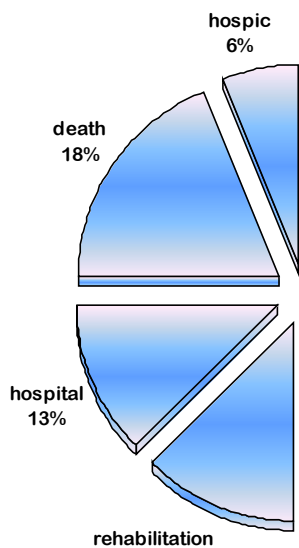

$13 \%$

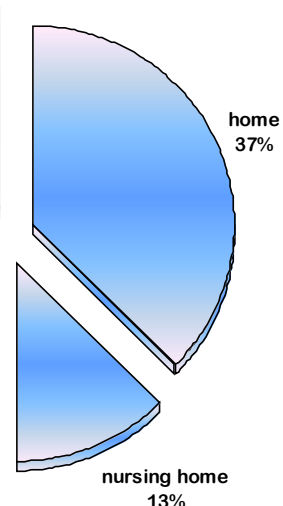

Figure 4: Transferring statistics after inpatient treatment.

a pneumonia with pneumothorax postoperatively. The overall complication rate was $37.5 \%$ and thus surgical complication rate $13.3 \%$.

\section{Discussion}

The 1-year survival rates of patients with spinal metastases depend on the primum and lies between 10 and 50\% [20,21]. In patients with paraplegia the 1-year survival rate is under $10 \%$ [8]. Therefore crucial for this group of patients is a supportive, quality of life-sustaining therapy. In the comparative analysis of our results with the current recommendation to treat metastatic related spinal cord injury, we are convinced that these patients do not have fixed guidelines or treatment recommendation compliance. The expected treatment benefit should be rather weighed against the risks of surgery and intensive care treatment [11]. However significant positive effects in terms of pain reduction, quality of life and attain long-term preservation of selfsufficiency could be observed based on our studies. Also patients with severe neurological deficits, who did not recover after surgery, benefited in regard to pain reduction. Thereby surgical treatment timing is significant for neurological outcome.

Thus, in a beginning paraplegia symptom an urgent surgical care should be sought within 24 hours. Also depending on the overall condition, patients should be timely operated within 48 hours after admission [22]. Despite controversy regarding the injection of methylprednisolon after NASCIS scheme, we think the indication for preoperative and postoperative dose given over 24 hours is given $[8,23]$. The surgical procedure in the case of metastasis-related acute paraplegic symptoms should be restricted to the timely and most gentle version of decompression and stabilization. Our research shows that patients are often in poor general condition. In addition to an acute, through metastasis caused, paraplegia there is a lack of time to carry out a comprehensive preoperative staging. Nevertheless, when spinal decompression is timely done, a regression of the neurological deficits can be achieved.

As surgical techniques are mono- or bisegmental laminectomy or laminoplasty. The reduction of a deformity and stabilization of the thoracic and lumbar spine is usually done by a dorsal fixation with a locking screw/rod system. If necessary, a mono- or multisegmental vertebral body resection is done with vertebral body replacement using bone graft or cage and augmentation with bone cement [24,25]. Postoperative application of the intensity-modulated radiotherapy, radionuclide therapy, androgen suppression or application of bisphosphonates should be decided in the interdisciplinary tumor conference [26,27]. Radiotherapy alone shows neither advantage in terms of neurological outcome nor in pain reduction. Thus it should be considered in inoperable patients and should vary in terms of tumorgenesis [12].

Decisive factors for the outcome and increase of quality of life are not only relieve of pain but timely mobilization and maintain or restore individual autonomy $[20,28]$. We could prove that it is possible to reduce pain through appropriate therapy for metastatic related acute paraplegia. In addition, a timely spinal decompression can improve neurological outcome [22]. Crucial to improving the neurologic symptoms is in addition to the surgical treatment, the consistent and timely physiotherapy and supporting these patients with appropriate technical aids [29].

To consolidate these results, it is essential to present patients postoperatively on an interdisciplinary tumor conference. Here can be set out the oncologic procedure of cancer, as well as additional surgery for tumor resection planned. The goal of the operation is prevention or elimination of instability and neurological deficits as well as adequate pain relief. Our studies show that all patients, regardless of initial neurological status, benefit from a post-operative pain relief.

Especially in spinal cord injury patients achieving a high degree of independence is essential. The control of bladder and bowel function should be achieved soon. Moreover, the transfer between wheelchair and bed should be trained. The home environment should be explored and if necessary adjusted. With intermittent self-catheterisation (ISK) or nurse-assisted Clinically Clean Intermittent Catheterisation (CCIC) urinary tract infections and pyelonephritis can be prevented. To prevent pressure ulcers, especially for the immunocompromised cancer patients, special positioning techniques should be used. Also thrombosis prevention and treatment of spasticity requires a proper therapy. Due to the complexity of the paraplegic aftercare, these rehabilitative measures should be initiated as soon as possible, at the same time offering advice and involving parents and relatives of the patients.

Not only the primary tumor but also the paraplegia is for the patient hard fate that changed the previous situation completely. We therefore consider it highly recommended that a psychological complementary treatment is included from the very beginning of the therapy. After initiation of these treatment measures we could discharge half of our patient's home, so that the other outpatient oncological therapy could be done with the support of family members. 2 of our patients were transferred to the oncological treatment in a specialized facility.

Due to the multi-morbidity of these patients in the studied group, a relatively high postoperative complication rate of $37.5 \%$ was found. Patients with metastatic tumors often exhibit a wide range of comorbidities. A significant influence on wound healing was observed 
for example by a poor nutritional status [30]. In our population of patients surgical complication rate was $13.3 \%$. Material failure or the necessity for surgical revision of internal fixation is a relatively rare complication [21].

\section{Conclusion}

In Summary, spinal metastases are an indication for surgical treatment, especially when they cause neurological deficits. The urgency of the operation depends on the status of metastasis, Tomita-type, Tomita-score, the risk of surgery, coagulation disorder, pain and the progression of neoplastic disease. Nevertheless, a complete paraplegic symptom should be relieved quickly surgically [31,32]. The natural course of the tumor disease cannot be affected by a palliative operation on the spine. For this reason, conservative and adjuvant measures should also be considered. With an appropriate therapy of metastatic related acute paraplegia, pain reduction and the neurological outcome improvement can be achieved. Here is essential besides the operation, consistent and timely physiotherapy and an appropriate medical aid supply $[12,26]$. A neurological deficit may be the first manifestation of a tumor disease. Symptoms like over months existing back pain, bladder and bowel dysfunction and a gait disorders in an often slow progressive tumors indicate an impending paraplegia. These symptoms should also be detected in patients with known prostate or breast cancer as hint of spinal metastases for a proper treatment to minimize the consequences of paraplegia. During this acute treatment a tumor staging is done by intraoperative biopsy and histology, tumor marker detecting and staging-CT $[15,24]$. As part of an interdisciplinary case conference the treatment regimen of the tumor should be specified ahead of the paraplegia treatment. The treatment must be individually adjusted according to the patient's general health, life expectancy, the primary tumor, metastasis and extent of neurological deficit. In any case, before any invasive therapy, specialized paraplegic centers should be contacted, to provide an optimal paraplegic and oncological treatment as well as supply of medical aids $[20,28]$.

\section{Conflict of Interests}

Authors have no conflict of interests to disclose.

\section{Reference}

1. George R, Jeba J, Ramkumar G, Chacko AG, Leng M, et al. (2008) Interventions for the treatment of metastatic extradural spinal cord compression in adults. Cochrane Database Syst Rev: CD006716.

2. Schultheiss M, Kinzl L, Hartwig E, Aarand M, David A (2007) Operative Therapieoption bei Skelettmetastasen. Orthopädie und Unfallchirurguie up2date 2: 141-156.

3. Quan GM, Vital JM, Pointillart V (2011) Outcomes of palliative surgery in metastatic disease of the cervical and cervicothoracic spine. J Neurosurg Spine 14: 612-618.

4. Schulte M, Schultheiss M, Hartwig E, Wilke HJ, Wolf S, et al. (2000) Vertebral body replacement with a bioglass-polyurethane composite in spine metastases-clinical, radiological and biomechanical results. Eur Spine J 9: 437-444

5. Winterer JT, Ghanem N, Uhl M, Langer M (2000) Radiologische Diagnostik: Skelettäre Metastasen und extraossäre Tumorausdehnung differentialdiagnostisch beurteilen. Klinikarzt 9: 281-284.

6. American Spinal Injury Assosciation. International Standards for Neurological Classification of Spinal Cord Injury, revised 2002. Chicago: American Spinal Injury Association; 2002.

7. Frankel HL, Hancock DO, Hyslop G, Melzak J, Michaelis LS, et al. (1969) The value of postural reduction in the initial management of closed injuries of the spine with paraplegia and tetraplegia. I. Paraplegia 7: 179-192.

8. Abel R (2003) Wirbelsäulenmetastasen und Querschnittlähmung. Orthopäde 32: $439-451$

9. Bartels RH, van der Linden YM, van der Graaf WT (2008) Spinal extradural metastasis: review of current treatment options. CA Cancer J Clin 58: 245-259.

10. Schiff D, O'Neill BP, Suman VJ (1997) Spinal epidural metastasis as the initial manifestation of malignancy: clinical features and diagnostic approach. Neurology 49: 452-456.

11. Costachescu B, Popescu CE (2010) Modern management in vertebral metastasis. Rom. Nsurg 17: 432-437.

12. Patchell RA, Tibbs PA, Regine WF, Payne R, Saris S, et al. (2005) Direct decompressive surgical resection in the treatment of spinal cord compression caused by metastatic cancer: a randomised trial. Lancet 366: 643-648.

13. Tokuhashi Y, Matsuzaki H, Kawano H, Sano S (1994) [The indication of operative procedure for a metastatic spine tumor: a scoring system for the preoperative evaluation of the prognosis]. Nihon Seikeigeka Gakkai Zasshi 68: 379-389

14. Hanbali F, Fourney DR, Marmor E, Suki D, Rhines LD, et al. (2002) Spinal cord ependymoma: radical surgical resection and outcome. Neurosurgery 51: 1162-1172.

15. Hirabayashi H, Ebara S, Kinoshita T, Yuzawa Y, Nakamura I, et al. (2003) Clinical outcome and survival after palliative surgery for spinal metastases: palliative surgery in spinal metastases. Cancer 97: 476-484.

16. Rosamond W, Flegal K, Furie K, Go A, Greenlund K, et al. (2008) Heart disease and stroke statistics--2008 update: a report from the American Heart Association Statistics Committee and Stroke Statistics Subcommittee. Circulation 117: e25-146.

17. Saklad M (1941) Grading of patients for surgical procedure. Anesthesiology 2: 281-284.

18. Tomita K, Kawahara N, Kobayashi T, Yoshida A, Murakami H, et al. (2001) Surgical strategy for spinal metastases Spine (Phila Pa 1976) 26: 298-306.

19. Homagk L, Hofmann GO, Zaage J, Stuttmann R (2012) A modular concept in postoperative pain management - interdisciplinarily and patient controlled. Trauma und Berufskrankheit.

20. Fattal C, Fabbro M (2011) Metastatiic Paraplegia an Functional Outcomes: Perspectives and Limitation for Rehabilitation Care. Part 1. Arch Phys Med Rehabil 92: 125-133

21. Wise JJ, Fischgrund JS, Herkowitz HN, Montgomery D, Kurz LT (1999) Complication, survival rates, and risk factors of surgery for metastatic disease of the spine. Spine (Phila Pa 1976) 24: 1943-1951.

22. Fürstenberg CH, Wiedenhöfer B, Gerner HJ, Putz C (2009) The effect of early surgical treatment on recovery in patients with metastatic compression of the spinal cord. J Bone Joint Surg Br 91: 240-244

23. Bracken MB, Shepard MJ, Collins WF, Holford TR, Young W, et al. (1990) A randomized, controlled trial of methylprednisolone or naloxone in the treatment of acute spinal-cord injury. Results of the Second National Acute Spinal Cord Injury Study. N Engl J Med 322: 1405-1411.

24. Jansson KA, Bauer HC (2006) Survival, complications and outcome in 282 patients operated for neurological deficit due to thoracic or lumbar spinal metastases. Eur Spine J 15: 196-202.

25. Simmons ED, Zheng $Y$ (2006) Vertebral tumors: surgical versus nonsurgical treatment. Clin Orthop Relat Res 443: 233-247.

26. Pilge H1, Holzapfel BM, Prodinger PM, Hadjamu M, Gollwitzer H, et al. (2011) [Diagnostics and therapy of spinal metastases]. Orthopade 40: 185-193.

27. O'Toole JE, McCormick PC (2003) Midline ventral intradural schwannoma of the cervical spinal cord resected via anterior corpectomy with reconstruction: technical case report and review of the literature. Neurosurgery 52: 1482-1485.

28. Fattal C, Fabbro M, Rouays-Mabit H, Verollet C, Bauchet L (2011) Metastatic paraplegia and functional outcomes: perspectives and limitations for rehabilitation care. Part 2. Arch Phys Med Rehabil 92: 134-145.

29. Rompe JD, Hopg CG, Eysel P (1999) Outcome after palliative posterior surgery for metastatic disease of the spine - evaluation of 106 consecutive patients after decompression an stabilisation with the Cortel-Dubousset instrumentation Arch Ortop Trauma Surg; 119: 394-400.

30. Pascal-Moussellard H, Broc G, Pointillart V, Siméon F, Vital JM, et al. (1998) Complications of vertebral metastasis surgery. Eur Spine J 7: 438-444.

31. North RB, LaRocca VR, Schwartz J, North CA, Zahurak M, et al. (2005) Surgical management of spinal metastases: analysis of prognostic factors during a 10 year experience. J Neurosurg Spine 2: 564-573.

32. Tan M, New $P$ (2011) Survival after rehabilitation for spinal cord injury due to tumor: a 12-year retrospective study. J Neurooncol 104: 233-238. 